\title{
Shadowgraphic imaging of carbon nanotube suspensions in water and in chloroform
}

\author{
L. Vivien, J. Moreau, and D. Riehl \\ Délégation Générale pour l'Armement, Centre Technique d'Arcueil, Department Laser, Optique et Thermo-Optique, \\ Arcueil, France \\ P. A. Alloncle and M. Autric \\ Institut de Recherche sur les Phenomenes Hors Equilibre, Marseille, France \\ F. Hache \\ Laboratoire d'Optique et Biosciences, Unité Mixte de Recherche, Centre National de la Recherche Scientifique 7645, \\ Ecole Polytechnique, Palaiseau, France \\ E. Anglaret \\ Groupe de Dynamique des Phases Condensées, Unité Mixte de Recherche, Centre National de la Recherche \\ Scientifique 5581, Université de Montpellier II, Montpellier, France
}

Received February 13, 2002; revised manuscript received June 14, 2002

\begin{abstract}
Carbon nanotube suspensions are known to display interesting optical limiting properties as a result of the formation of solvent or carbon-vapor bubbles that scatter the laser beam. In this study we present an original experiment that permits direct observation of the changes that occur in the focal zone in carbon nanotube suspensions by using a shadowgraphic experiment coupled with a monochromatic pump-probe experiment. We have observed a clear correlation between the radius of the scattering centers and the evolution in transmittance of the sample. We compared bubble growth in chloroform and water and found good agreement with previously obtained results. We also observed the presence of compression waves, which propagate parallel to the laser beam and can produce secondary cavitation phenomena after reflection on the cell walls. (C) 2002 Optical Society of America
\end{abstract}

OCIS codes: $190.3970,190.4400,190.4870,290.5850$.

\section{INTRODUCTION}

The proliferation of lasers and systems that employ lasers has brought with it the danger of adverse effects from these bright, coherent light sources. These effects include the possibility of damage from pulsed lasers as well as of temporary blinding by continuous-wave lasers. With nearly every possible wavelength being emitted by these sources, there exists a need to develop optical limiters and tunable filters that can suppress undesired radiation at any wavelength. Nowadays, laser sources (optical parametric oscillators, dye lasers, Raman lasers,...) are widely used in many applications, not only in research laboratories but also in large areas of industry and medicine and for military applications, and they pose a potential hazard to eyes and other optical sensors (CCDs, thermal cameras, ...). These developments have motivated extensive research activity to design optical limiting systems for eye and sensor protection. ${ }^{1,2}$ An ideal optical limiter is a device for which, at low input fluence, the linear transmittance is high $(>70 \%)$, whereas, at high input fluence, the output energy is below the damage threshold of sensors or eyes. The device's damage threshold must be high, as must be the limiting threshold. Nonlinear optical limiters may protect optical sensors or eyes against pulsed lasers of any wavelength (from visible to infrared radiation) and pulse durations (from a few picoseconds to continuous waves). To this end, all existing nonlinear materials require a tightly focused beam for initiating the effect and must therefore be incorporated into optical systems. Several nonlinear effects have been proposed: nonlinear absorption (reverse saturable absorption, ${ }^{3}$ multiphoton absorption), nonlinear refraction (electronics ${ }^{4}$ or thermal effects ${ }^{5}$ ), and nonlinear scattering (solvent bubble formation, or particle sublimation, or both ${ }^{6-8}$ or mismatched indices ${ }^{9}$ ). All these processes exhibit optical limiting behavior with advantages and disadvantages.

To achieve optical limiting we used carbon nanotubes suspended in water and in chloroform. In previous studies, ${ }^{10-13}$ some of the present authors and others determined that the optical limiting effect in these materials originates in the growth of scattering centers as a result of two physical mechanisms: The first one is thermal transfer from the particles to the surrounding liquid, and the second is sublimation of the carbon nanotubes, which lead to solvent and carbon-vapor bubble growth, respectively. These gaseous cavities scatter the laser beam, leading to a drop in the sample transmittance. Carbon nanotube suspensions demonstrate good 
efficiency for wavelengths from 400 to $1100 \mathrm{~nm}$ for pulse widths from 3 to $100 \mathrm{~ns}$ (Ref. 12) and possibly for pulse widths up to several microseconds. ${ }^{14}$ In addition, we have been able to measure the average radius and the concentration of scattering centers by carrying out a polychromatic pump-probe experiment and using a theoretical model that couples the Beer-Lambert law and Mie theory. ${ }^{13}$ These experiments have shown that nonlinear scattering is due to many small vapor bubbles in chloroform and to a small number of large vapor bubbles in water suspensions. We also observed a stronger coalescence in water than in chloroform. Measuring the evolution of scattering centers and the vapor bubble distribution during and after the pump pulse, we observed a coalescence effect $^{13}$ and a cavitation phenomenon in the microsecond regime. ${ }^{10}$ In this paper we present new results that we obtained with a shadowgraphic experiment. This experiment consists in the direct photography of scattering centers by means of an ultrarapid camera coupled with a traditional pump-probe experiment. This kind of experiment has been widely used to illustrate plasma, bubble, and shock-wave formations that results from laser-induced breakdown in aqueous media. ${ }^{15}$ Goedert et al. adapted this experiment for optical limiters including nonlinear scattering materials. ${ }^{16}$ We have also used the technique to gain a better understanding of vapor bubble growth and have added a pump-probe experiment to measure the transmittance perturbation simultaneously.

In Section 2 we present a synthesis method and a purification procedure for single-wall carbon nanotubes. The shadowgraphic and pump-probe experimental setup is described in Section 3. In Section 4 we report the results obtained $150 \mathrm{~ns}$ to $11 \mu \mathrm{s}$ after the pump pulse in carbon nanotube suspensions in chloroform. We compare the evolution of the probe transmittance and the scattering centers in these suspensions. We also compare the difference between chloroform and water suspensions to confirm the results obtained in a previous study of the wavelength dependence of scattering efficiency. ${ }^{13}$ Among the various mechanisms for the nonlinear scattering in carbon black and carbon nanotube suspensions, the formation of a microplasma has sometimes been proposed. In this study we determine the input energy for which plasma is formed in the focal zone; we have been able to determine the effect that is responsible for the optical limiting properties in carbon nanotube suspensions. Finally, in Section 5 we present results obtained with an intensified CCD camera in which we observed the kinetics of vapor bubble growth from 5 to $150 \mathrm{~ns}$ after the maximum of the pump pulse.

\section{CARBON NANOTUBE SUSPENSIONS}

Carbon nanotubes were synthesized by the electric arc discharge technique. ${ }^{17}$ An electric arc discharge is created between two graphite electrodes in a helium atmosphere in the presence of a metallic catalyst $(\mathrm{Ni}$ and $\mathrm{Y}$ at 4.2:1 at. \%) for the synthesis of single-wall carbon nanotubes (SWNTs). As-prepared samples contain impurities such as amorphous carbon, graphite, fullerenes, and residual catalysts. The SWNT samples are purified in a three-step procedure. ${ }^{18}$ The first step uses a nitric acid treatment at $100{ }^{\circ} \mathrm{C}$ that partially disintegrates the catalyst particles and disentangles the complex network of nanotubes, other carbonous materials, and catalyst particles. The second step is a tangential filtration that allows the nanotubes to be separated from the other species. Finally, a thermal treatment in an inert atmosphere eliminates residual catalysts. After such purification, the amount of SWNT is close to $90 \mathrm{vol} . \%$ in the samples. SWNTs usually self-assemble on a triangular array into crystalline nanobundles of a couple to some tens of tubes. ${ }^{19}$ The tubes are 1.3-1.5 nm in diameter $^{17,19}$ and are several micrometers long. Singlewall carbon nanotube suspensions in water and in chloroform are tested in $2-\mathrm{mm} \times 2-\mathrm{mm}$ square cells. The linear transmittance is larger than $70 \%$ at $532 \mathrm{~nm}$. The water (chloroform) suspensions are stable without any stirring for 6 (1) months.

\section{EXPERIMENTAL SETUP}

The aim of the experiment is to associate shadowgraphic illustration with a pump-probe experiment to connect the evolution of the gaseous cavities created by the pump laser in a carbon nanotube suspension with the change of transmittance of the probe. Figure 1 presents the experimental setup. The pump beam is directly obtained from a horizontally polarized Nd:YAG laser delivering 13-ns FWHM pulses at 532 or $1064 \mathrm{~nm}$. To eliminate the cumulative effect we use this laser in single-shot mode. This monochromatic pump-probe configuration is similar to one used previously. ${ }^{10}$ The input energy is recorded with a pyroelectric detector, and the temporal profile is recorded with a fast Si sensor. The pump beam passes through a polarizing cube to preserve horizontal polarization and is focused into the sample with a $200-\mathrm{mm}$ focallength achromatic doublet ( $\sim f / 20$ focusing geometry). The sample is probed at $633 \mathrm{~nm}$ by a continuous $\mathrm{He}-\mathrm{Ne}$ laser beam that is vertically polarized to permit separation from the pump beam by a polarizing cube. The pump beam's waist into the sample is 20 and $50 \mu \mathrm{m}$ at 532 and $1064 \mathrm{~nm}$, respectively. The experimental configuration is chosen such that the probe's waist is always smaller than that of the pump.

To this pump-probe experiment we add a second probe beam delivered by an argon-ion laser at $488 \mathrm{~nm}$ that illuminates the carbon nanotube sample at $90^{\circ}$ to the pump's optical axis. With a $100-\mathrm{mm}$ focal-length lens we image the focal zone of the pump onto a camera. The bubble dynamics is investigated with a high-speed Imacon 790 (Hadland) camera. This camera permits framing rates of $10^{4}$ to $2 \times 10^{7}$ frames/s and streak velocities from 10 $\mu \mathrm{s} / \mathrm{mm}$ to $1 \mathrm{~ns} / \mathrm{mm}$. The first acquisition takes place $\sim 150 \mathrm{~ns}$ after the pump pulse. Thus we could not see the primary events described in Ref. 10, but we clearly observed cavitation phenomena, as previously observed in monochromatic and polychromatic pump-probe experiments. ${ }^{10,13}$ We can be more quantitative and compare the evolution of the probe transmittance and the vapor bubble radii measured in the shadowgraphic imaging.

To determine the kinetics of gaseous cavities we also use an intensified CCD camera which permits the evolu- 
tion of scattering centers to be observed on a nanosecond time scale (from 5 to $150 \mathrm{~ns}$ ). We determine the evolution of vapor bubbles in the focal zone for times as short as $5 \mathrm{~ns}$, i.e., during the pump pulses at $1064 \mathrm{~nm}$. This experiment is complementary to the experiment described above. Both experiments make it possible to follow the evolution of the bubbles from the first moments to $11 \mu$ s after they begin to form.

\section{MICROSECOND TIME-SCALE RESULTS}

\section{A. Time Evolution}

In the first study we measure with the Imacon camera the evolution of scattering centers in carbon nanotube suspensions. We observe cavitation phenomena, and we compare the evolution of the probe transmittance and of the bubble radius. Figure 2 shows the temporal evolution of scattering centers for carbon nanotube suspensions in chloroform for an input fluence of $9 \mathrm{~J} / \mathrm{cm}^{2}$ at 532 $\mathrm{nm}$. Twenty-two images were acquired every $500 \mathrm{~ns}$, and the first was saved $150 \mathrm{~ns}$ after the pump pulse. Each image scans an area of $425 \mu \mathrm{m} \times 375 \mu \mathrm{m}$, corresponding to a quarter of the cell thickness. Simultaneously, we measure the probe perturbation with the pump-probe experiment. A cloud of small vapor bubbles is observed in the focal zone created by interaction between the laser beam and the suspensions. The peripheral bubbles are visible and seem homogeneous. The central part of the bubble cloud is not resolved by our imaging system. We observe a gradual diminution of the bubble cloud, and the small gaseous cavities located on the periphery of the cloud disappear rapidly, leading to a strong concentration of vapor bubbles in the central zone. Then the bubble cloud decreases gradually until it appears as shown in the photo numbered 16, where it experiences a new expansion phase that corresponds to a cavitation rebound. From these pictures we can conclude that, in chloroform, cavitation is not produced by a single macroscopic bubble but is due to the oscillation in phase of microscopic bubbles, giving rise to a collective effect.

In each image of Fig. 2 we measure the transversal semisection of the bubble cloud, which can be associated with an effective radius of scattering centers. Figure 3 presents the kinetics of this effective radius and the probe transmittance. The evolutions of the effective radius and of the probe transmittance are similar. The radius rapidly reaches $60 \mu \mathrm{m}$ and then collapses in $9 \mu \mathrm{s}$ to a minimum of $5 \mu \mathrm{m}$ before rebounding to $20 \mu \mathrm{m}$ at the first rebound. Concomitantly, the initial transmittance drop is followed by a partial recovery when the radius is minimum and by a further drop when the radius increases. The similarity between the two curves is striking, confirming clearly the role of the laser-induced gaseous cavities in the scattering process. Considering now the decrease in bubble radius after the first microsecond, we can understand it as a competition between bubble expansion and the pressure effect of the surrounding solvent. Some of the present authors have already performed such a study by implementing an analysis based on the Rayleigh-Plesset model. ${ }^{10,11}$ Here we get a nice confirmation of that study: Indeed, considering the experimental rebound time of $8 \mu$ s (see Fig. 3), the model indi- cates that the maximum radius is $65 \mu \mathrm{m}$, in very good agreement with our measurements.

By increasing the input fluence to $50 \mathrm{~J} / \mathrm{cm}^{2}$ we were able to observe plasma formation at the entrance of the cell. Figure 4(a) shows the image sequences at $50 \mathrm{~J} / \mathrm{cm}^{2}$. Two features are worth noting. First, one can observe bursts of matter that escape from the vapor bubble created by the laser beam. Second, there are bright filaments inside the gaseous cavity, which seem to emanate from the side and attest to the presence of a plasma. Streak acquisition permits a better quantification of the expansion kinetics of the gaseous cavity [Fig. 4(b)] at 26 $\mathrm{J} / \mathrm{cm}^{2}$. At $T_{0}$ we observe a shock wave following the initial breakdown in the media. The velocity measured corresponds exactly to the sound velocity of $990 \mathrm{~m} / \mathrm{s}$ in chloroform. We can also measure the velocity of the cavity expansion. Initially this velocity is approximately 150 $\mathrm{m} / \mathrm{s}$; it decreases rapidly to $40 \mathrm{~m} / \mathrm{s}$ after $5 \mu \mathrm{s}$. From these experiments it is not possible to know whether the plasma formation is a phenomenon that follows carbon nanotube sublimation or whether it emanates from the wall of the cell, as the laser beam is tightly focused from the beginning of the cell. Whatever its origin, the plasma formation induces damage in the optical limiter device and is responsible for the optical damage threshold.

To compare the bubble evolutions of the chloroform and the water suspensions we performed these measurements in the same experimental conditions for both solvents. Figure 5 shows two sequences obtained with carbon nanotubes suspended in chloroform and in water for an input fluence of $9 \mathrm{~J} / \mathrm{cm}^{2}$ at $532 \mathrm{~nm}$. The behavior of water suspensions clearly differs from that of chloroform suspensions, with better coalescence for water suspensions. Starting from the first image, $\sim 150 \mathrm{~ns}$ after the pump pulse the vapor bubbles are less numerous and are approximately twice as large (radii of $10 \mu \mathrm{m}$ for water and 5 $\mu \mathrm{m}$ for chloroform). For water suspensions, we observe after image 1, two big vapor bubbles that oscillate in phase. These results are in agreement with the theoretical scattering model developed by coupling of the BeerLambert law and Mie theory with polychromatic pumpprobe experiments. ${ }^{13}$ Indeed, from this analysis we determined the evolution of the concentration and average radius of the scattering centers as well as their volume fraction and observed a slower coalescence in chloroform suspensions than in water. Moreover, we have demonstrated that scattering in chloroform is due to a large number of small vapor bubbles, whereas in water it is due to a small number of large vapor bubbles.

\section{B. Energy Dependence}

In Subsection 4.A we studied the evolution of scattering centers to determine the kinetics of the growth of vapor bubbles in carbon nanotube suspensions in water and in chloroform. Here we are interested in the effects of the input fluence on the number, size, and distribution of gaseous cavities in the samples. Figure 6 presents, for both suspensions, the images that were saved $\sim 150 \mathrm{~ns}$ after the pump pulse for different input fluences. First, we can observe the large number of vapor bubbles in chloroform suspensions. Second, the size of an individual cav- 


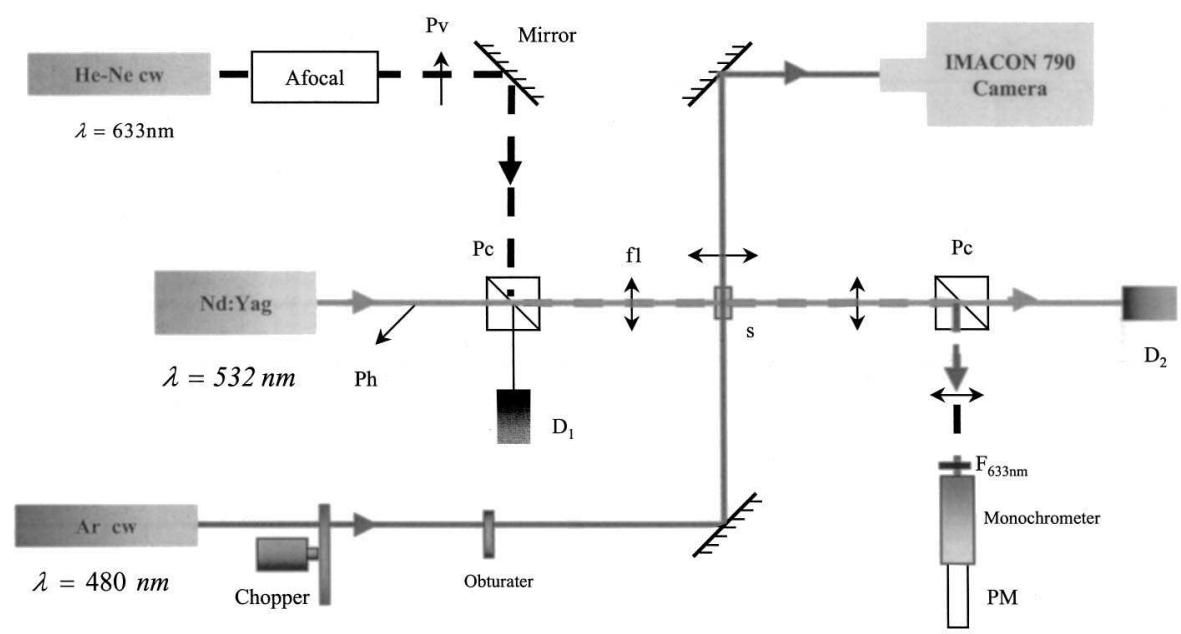

Fig. 1. Shadowgraphic experimental setup coupled with a classic pump-probe experiment with a continuous-wave He-Ne laser: Pc's, polarizer cubes; PT, fast photocathode; PM, photomultiplier; $\mathrm{Pv}, \mathrm{Ph}$, vertical and horizontal polarization, respectively; f1, 200-mm focallength achromatic doublet; s, sample; $\mathrm{D}_{1}, \mathrm{D}_{2}$, input and output photodetectors, respectively; Afocal, a focal lens.

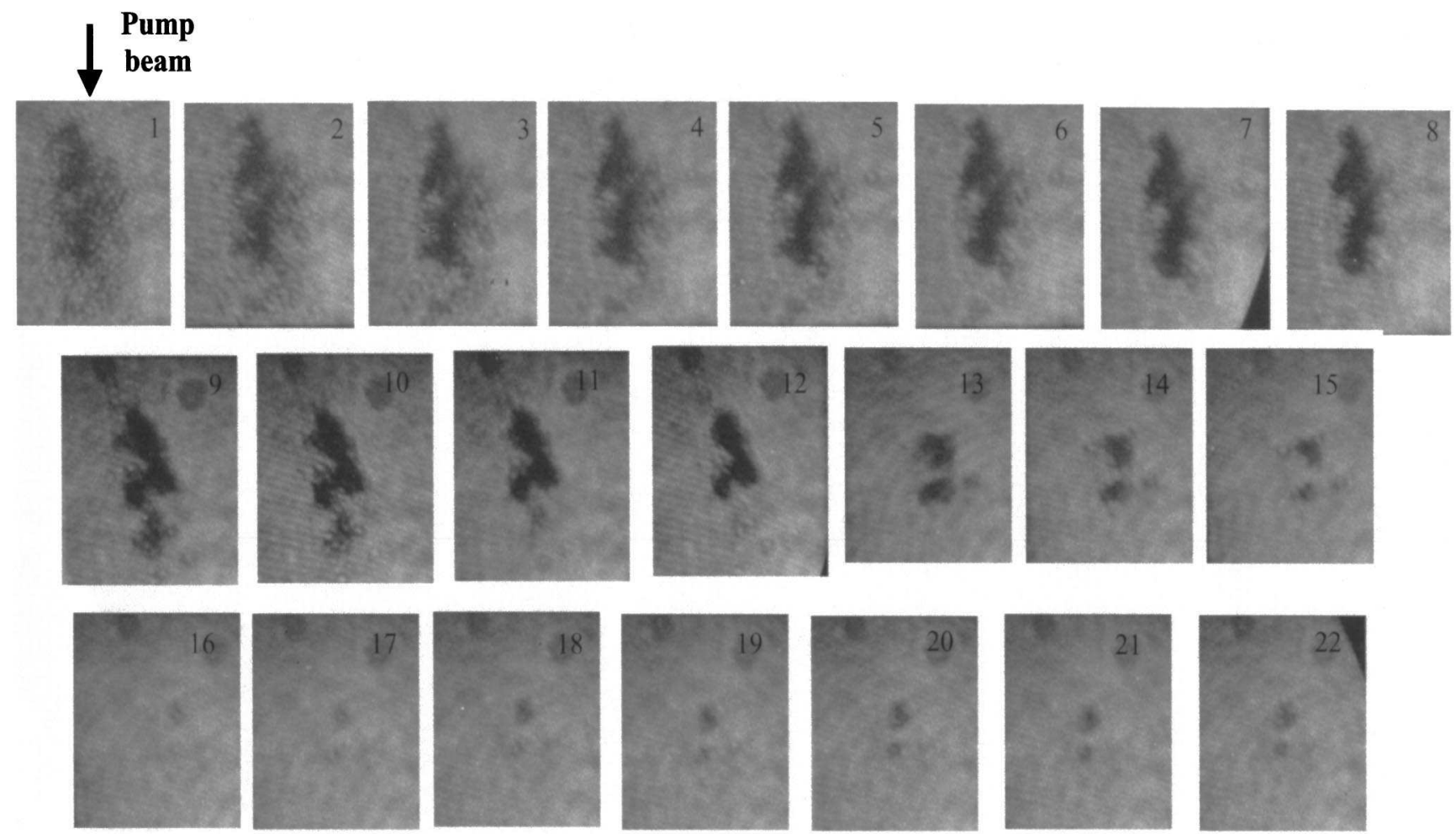

Fig. 2. Illustration of the evolution of solvent vapor bubble growth for carbon nanotube suspensions in chloroform. The images are separated by $500 \mathrm{~ns}$, and image 1 is obtained $\sim 150 \mathrm{~ns}$ after the maximum of the input pump pulse. The input fluence at $532 \mathrm{~nm}$ is 9 $\mathrm{J} / \mathrm{cm}^{2}$.

ity seems to be independent of the pump fluence, which means that a coalescence phenomenon already occurred earlier than $150 \mathrm{~ns}$ after the maximum of the input pump pulse.

At low input fluences of 2 and $4.5 \mathrm{~J} / \mathrm{cm}^{2}$ in carbon nanotube suspensions in chloroform and in water, respectively, the vapor bubble distribution fits the focal volume of the laser beam. However, at high input fluences this distribution largely overflows the focal volume. The beam waist is $20 \mu \mathrm{m}$, whereas the cloud radius of vapor bubbles varies from $\sim 18 \mu \mathrm{m}$ at $350 \mathrm{~mJ} / \mathrm{cm}^{2}$ to $\sim 90 \mu \mathrm{m}$ at $15 \mathrm{~J} / \mathrm{cm}^{2}$ in chloroform. For water suspensions the cloud radius of gaseous cavities varies from $12 \mu \mathrm{m}$ at $1.5 \mathrm{~J} / \mathrm{cm}^{2}$ to $70 \mu \mathrm{m}$ at $22 \mathrm{~J} / \mathrm{cm}^{2}$. This variation can be understood as follows: For low input fluences the interaction with the laser beam occurs over the entire focal zone, as is clearly observable from images numbered 7 and 8 in Fig. 5 for both solvents, whereas at high input fluence there is a large number of bubbles generated out of the focal volume. The presence of bubbles outside the laser interaction zone may have either of two causes: The bubbles can be new ones created by the laser light scattered outside the focal volume, or 
they can come from motion of the original bubbles caused by thermal activity or electrostriction.

Note that we can observe vapor bubbles starting from $350 \mathrm{~mJ} / \mathrm{cm}^{2}$ and $1.5 \mathrm{~J} / \mathrm{cm}^{2}$ for chloroform and water suspensions, respectively. Below these input fluences we could not see gaseous cavities with our experimental magnification of $40 \times$. A reason for this is that the vapor bubble lifetimes are small near the optical limiting threshold, and it is probable that the sample perturbation finishes before the first acquisition begins for low energies.

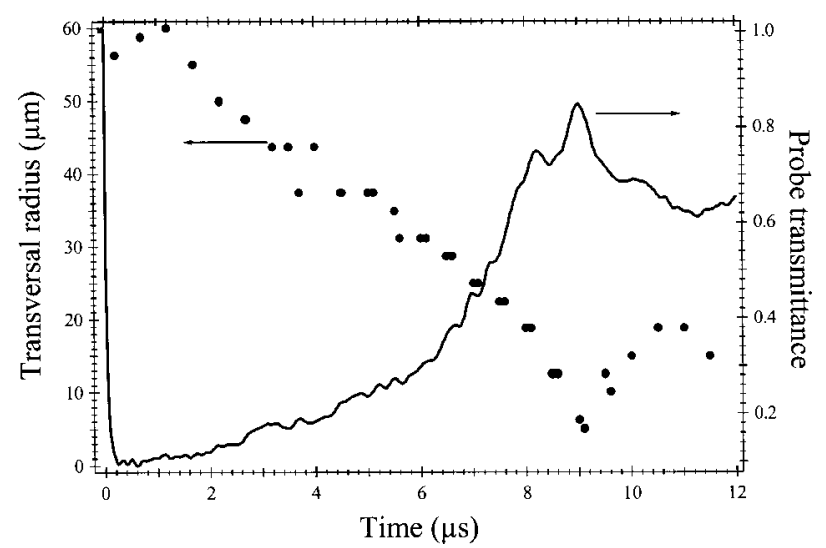

Fig. 3. Evolution of the probe transmittance and the transversal radius of the vapor bubble cloud for carbon nanotube suspensions in chloroform. Zero time corresponds to the maximum of the input pump pulse at $532 \mathrm{~nm}$. The input fluence was $9 \mathrm{~J} / \mathrm{cm}^{2}$ at $532 \mathrm{~nm}$.

\section{Compression Wave Effect}

For high input energy $\left(\sim 15 \mathrm{~J} / \mathrm{cm}^{2}\right)$ we notice a strange perturbation of the probe beam (Fig. 7). We can observe two cavitation oscillations that occur within $25 \mu$ s after the pump pulse, as already observed (cf. Fig. 3), but we can also observe two unexpected damped images that occur every $40 \mu \mathrm{s}$. We attribute these perturbations to the creation of a compression wave. In this hypothesis the time interval corresponds to the propagation of an acoustic wave that we can calculate to $\sim 40 \mathrm{~mm}$, considering that the velocity of sound in chloroform is $990 \mathrm{~m} / \mathrm{s}$. This result is consistent with the reflection of an acoustic wave created in the focal volume on the bottom of the cell, as, in our experiment, the pump and probe beams are approximately $20 \mathrm{~mm}$ above the wall of the cell. These perturbations of the probe transmittance last, however, for too long a time to result only from index modulation of the medium owing to the reflection of an acoustic wave. The refractive signature associated with such an effect would give a peak-to-valley or a valley-to-peak shape and would be faster. We therefore think that the reflection of the wave reactivates the cavitation phenomenon in the notyet-recondensed gaseous cavities.

\section{NANOSECOND TIME-SCALE RESULTS}

We made similar shadowgraphic experiments with an intensified CCD camera that allows one to acquire images of the focal volume from 5 to $150 \mathrm{~ns}$ after the pump pulse.
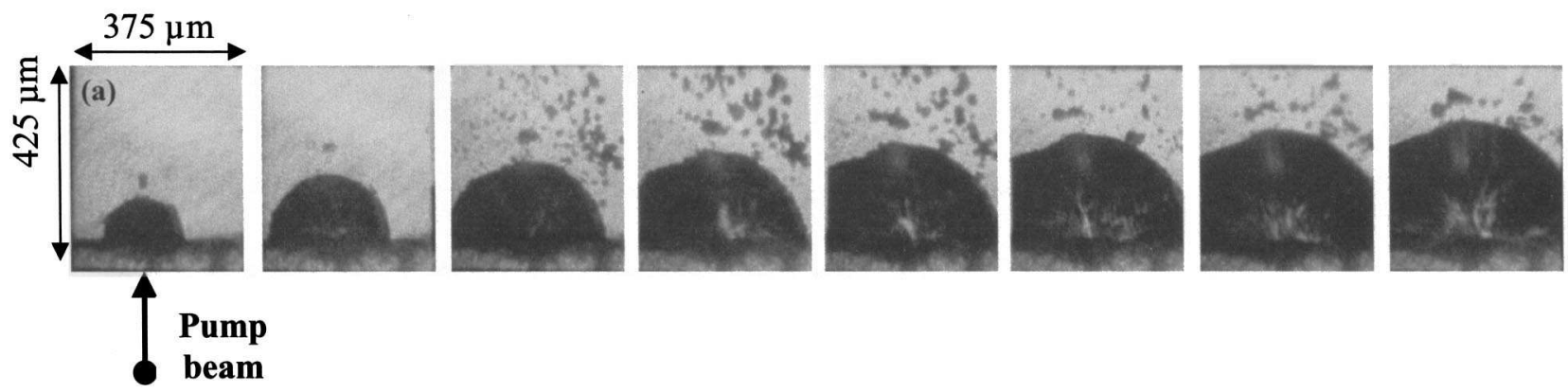

Pump
beam

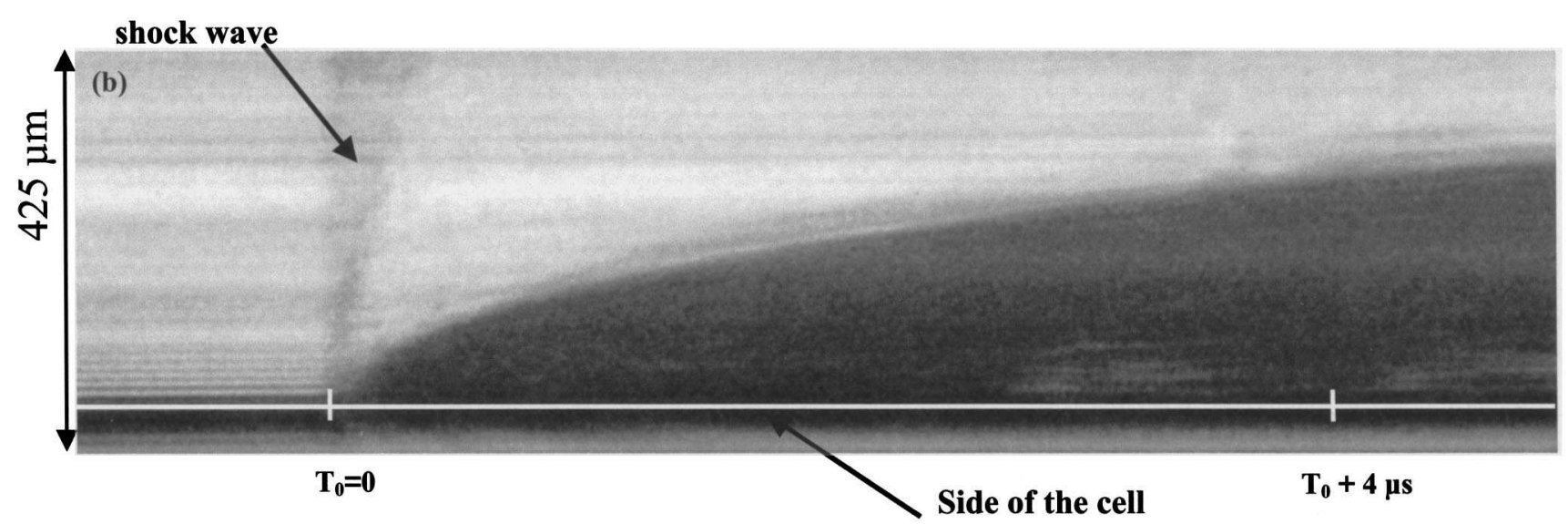

Fig. 4. (a) Formation and growth of plasma in carbon nanotube suspensions in chloroform. The input fluence is $50 \mathrm{~J} / \mathrm{cm}^{2}$ at $532 \mathrm{~nm}$. The images are separated by $500 \mathrm{~ns}$, and the first image (top left) was obtained $\sim 150 \mathrm{~ns}$ after the maximum of the input pump pulse. (b) Kinetics of plasma growth in carbon nanotube suspensions in chloroform in streak mode acquisition. The input fluence is $26 \mathrm{~J} / \mathrm{cm}^{2}$ at $532 \mathrm{~nm}$, and the streak velocity is $100 \mathrm{~ns} / \mathrm{mm}$. 


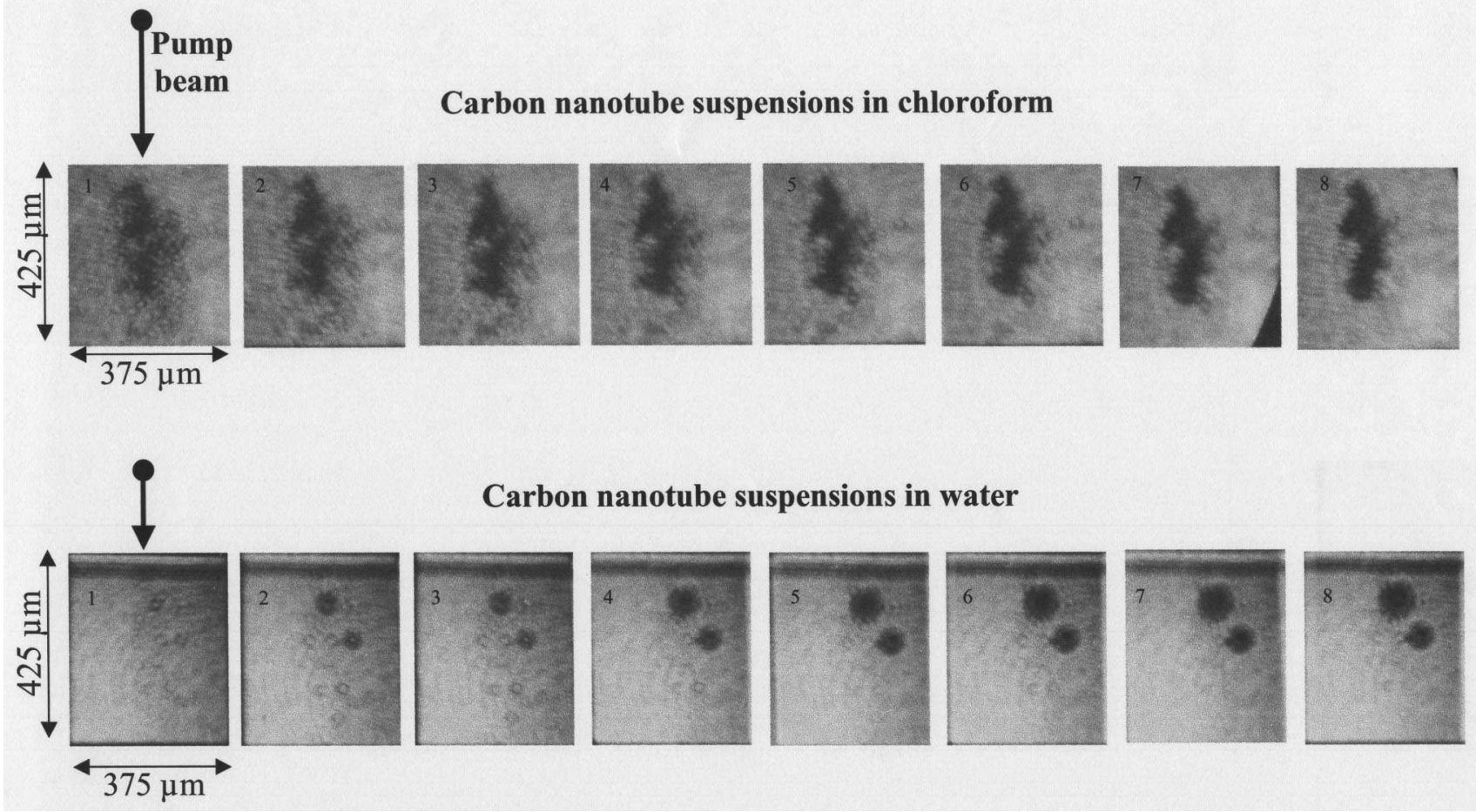

Fig. 5. Comparison of the evolution of the scattering centers for carbon nanotube suspensions in chloroform and in water. The input fluence is $9 \mathrm{~J} / \mathrm{cm}^{2}$ at $532 \mathrm{~nm}$, and the pulse duration $13 \mathrm{~ns}$. The images are separated by $500 \mathrm{~ns}$, and image 1 is obtained $\sim 150 \mathrm{~ns}$ after the maximum of the input pump pulse.

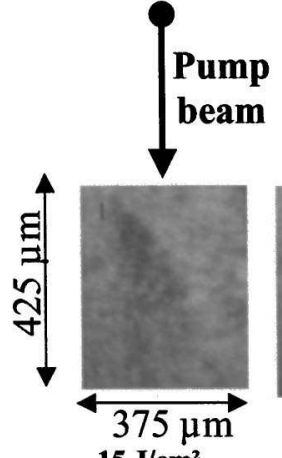

$15 \mathbf{J} / \mathbf{c m}^{2}$

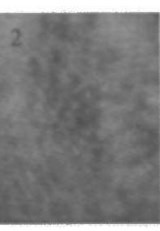

$9 \mathrm{~J} / \mathrm{cm}^{2}$

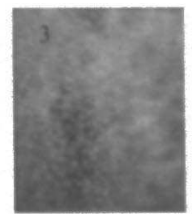

$4.6 \mathrm{~J} / \mathrm{cm}^{2}$

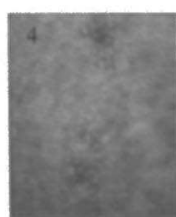

$3 \mathrm{~J} / \mathrm{cm}^{2}$

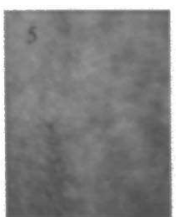

$2 \mathrm{~J} / \mathrm{cm}^{2}$

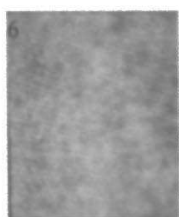

$860 \mathrm{~mJ} / \mathrm{cm}^{2}$

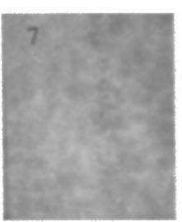

$450 \mathrm{~mJ} / \mathrm{cm}^{2}$

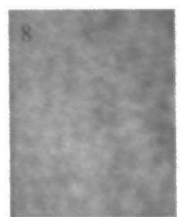

$350 \mathrm{~mJ} / \mathrm{cm}^{2}$

\section{Carbon nanotube suspensions in water}
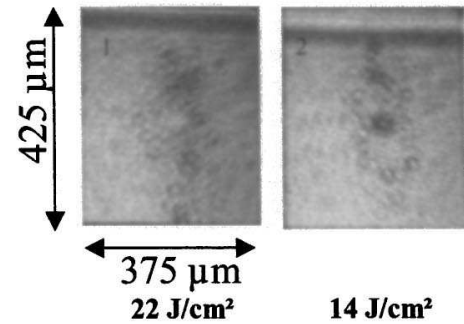

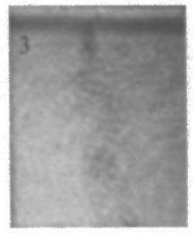

$8 \mathrm{~J} / \mathrm{cm}^{2}$

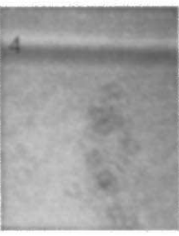

$7 \mathrm{~J} / \mathrm{cm}^{2}$

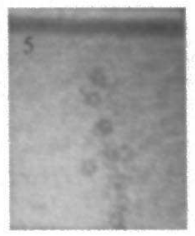

$4.5 \mathrm{~J} / \mathrm{cm}^{2}$

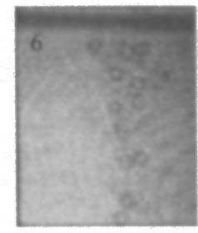

$3 \mathbf{J} / \mathrm{cm}^{2}$

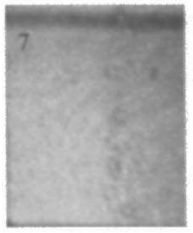

$1.8 \mathrm{~J} / \mathrm{cm}^{2}$

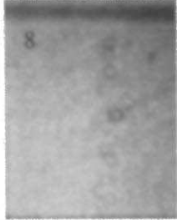

$1.5 \mathrm{~J} / \mathrm{cm}^{2}$

Fig. 6. Dependence on energy of the repartition of the scattering centers for carbon nanotube suspensions in chloroform (from 350 $\mathrm{mJ} / \mathrm{cm}^{2}$ to $15 \mathrm{~J} / \mathrm{cm}^{2}$ ) and in water (from 1.5 to $22 \mathrm{~J} / \mathrm{cm}^{2}$ ). The images are separated by $500 \mathrm{~ns}$, and image 1 is obtained $\sim 150 \mathrm{~ns}$ after the maximum of the input pump pulse.

These experiments were performed at $1064 \mathrm{~nm}$ to eliminate parasitic problems caused by the high sensitivity of the camera in the visible.
We observed the evolution of the vapor bubbles and the propagation of compression waves created by the pump beam. Figure 8 shows the evolution of bubble growth 


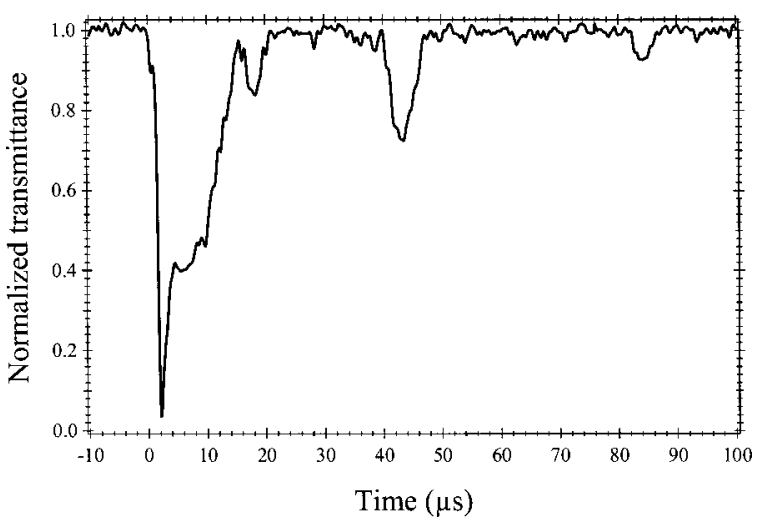

Fig. 7. Probe beam perturbation of carbon nanotube suspensions in chloroform on a microsecond time scale. The input fluence is $15 \mathrm{~J} / \mathrm{cm}^{2}$ at $532 \mathrm{~nm}$.

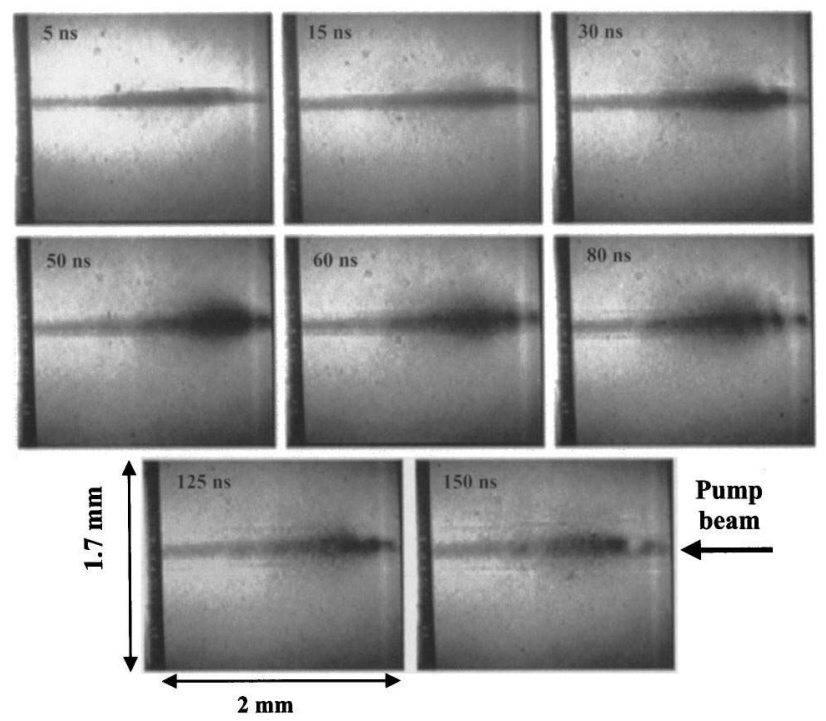

Fig. 8. Evolution of scattering centers during the pump pulse from 5 to $150 \mathrm{~ns}$ for carbon nanotube suspensions in chloroform. The input fluence is $9 \mathrm{~J} / \mathrm{cm}^{2}$ at $1064 \mathrm{~nm}$.

from 5 to $150 \mathrm{~ns}$ from the maximum of pump pulse. We measured one image by laser shot. In the first image the bubbles are confined to the focal volume. Then, as time goes on, we can clearly observe the escape of these bubbles out of the interaction zone, as we have already inferred from Fig. 6.

After image 5 we observe two dark horizontal faint streaks, which are due to acoustic-wave propagation in the medium. These acoustic waves are created by the intense pump pulse. They can be responsible for a part of the nonlinear refraction observed with 7 -ns pulse duration at 532 and $1064 \mathrm{~nm}^{20}$ It is the rebound of these acoustic waves that gives birth to the reappearance of the probe perturbation shown in Fig. 7.

\section{CONCLUSION}

In this paper we have described shadowgraphic experiments that have allowed us to illustrate the focal volume during and after the pump pulse in various suspensions of carbon nanotubes. We have also coupled classic pump- probe experiments with a continuous $\mathrm{He}-\mathrm{Ne}$ laser as a probe to associate the scattering centers with the probe perturbation.

We have observed, in carbon nanotube suspensions in chloroform on a microsecond time scale, the formation of microbubbles that oscillate in phase as the result of a cavitation phenomenon. In water suspensions an important coalescence effect leads to the formation of a few macroscopic vapor bubbles, whereas in chloroform suspensions we observe numerous microscopic gaseous cavities. This difference can be explained by the larger surface tension at the liquid-gas interface for water than for chloroform. This coalescence phenomenon is in agreement with a previous experiment in which the wavelength dependence of the scattered light was measured. ${ }^{13}$ We have also observed evidence of plasma generation in carbon nanotube suspensions in chloroform under high input fluences $\left(10-20 \mathrm{~J} / \mathrm{cm}^{2}\right)$.

At low input fluences the scattering centers are distributed in the focal volume of the pump beam, whereas at high input fluences the vapor bubbles overflow the beam caustic as a result of secondary excitation of nanotubes or of thermal or electrostrictive motion of the bubbles. This phenomenon occurs rapidly after the pump pulse $(\sim 20$ ns). We have also observed shock waves in carbon nanotube suspensions in chloroform, generating a surprising effect after beam propagation as an acoustic wave initiates a new cavitation phenomenon after reflection on the walls of the cell.

\section{ACKNOWLEDGMENTS}

The authors greatly appreciate the high-quality samples provided by S. Tahir and P. Bernier. We thank M. Andrieux and F. Lafonta for their help and for fruitful discussions. E. Anglaret acknowledges financial support from the Délégation Générale pour l’Armement, France.

\section{REFERENCES}

1. K. Nashimoto, R. Pachter, B. W. Wessels, J. Shmulovich, A. K.-Y. Jen, K. Lewis, R. Sutherland, and J. W. Perry, eds., Thin Films for Optical Waveguide Devices and Materials for Optical Limiting, Vol. 597 of MRS Proceedings Series (Materials Research Society, Warrendale, Pa., 1999).

2. R. C. Hollins, "Materials for optical limiters," Curr. Opin. Solid State Mater. Sci. 4, 189-196 (1999).

3. J. W. Perry, "Organics and metal-containing reverse saturable absorbers for optical limiters," in Nonlinear Optics of Organic Molecules and Polymers, H. S. Nalwa and S. Miyata, eds. (CRC Press, Orlando, Fla., 1997), pp. 813-840.

4. R. W. Boyd, Nonlinear Optics (Academic, New York, 1992).

5. B. L. Justus, A. L. Huston, and A. J. Campillo, "Broadband thermal optical limiter," Appl. Phys. Lett. 63, 1483-1486 (1993).

6. K. J. McEwan, P. K. Milsom, and D. B. James, "Nonlinear optical effects in carbon suspensions," in Nonlinear Optical Liquids for Power Limiting and Imaging, C. M. Lawson, ed., Proc. SPIE 3472, 42-52 (1998).

7. K. M. Nashold and D. P. Walter, "Investigations of optical limiting mechanisms in carbon particle suspensions and fullerene solutions," J. Opt. Soc. Am. B 12, 1228-1237 (1995).

8. K. Mansour, M. J. Soileau, and E. W. Van Stryland, "Nonlinear optical properties of carbon-black suspensions (ink)," J. Opt. Soc. Am. B 9, 1100-1109 (1992). 
9. V. Joudrier, P. Bourdon, F. Hache, and C. Flytzanis, "Characterization of nonlinear scattering in colloidal suspensions of silica particles," Appl. Phys. B 70, 105-109 (2000).

10. L. Vivien, D. Riehl, E. Anglaret, and F. Hache, "Pumpprobe experiments at $1064 \mathrm{~nm}$ in singlewall carbon nanotube suspensions," IEEE J. Quantum Electron. 36, 680-686 (2000).

11. L. Vivien, D. Riehl, F. Hache, and E. Anglaret, "Nonlinear scattering origin in carbon nanotube suspensions," J. Opt. Nonlin. Phys. Mater. 9, 297-308 (2000).

12. L. Vivien, D. Riehl, P. Lançon, F. Hache, and E. Anglaret, "Pulse duration and wavelength effects on the optical limiting behavior of carbon nanotube suspensions," Opt. Lett. 26, 26-29 (2001).

13. L. Vivien, J. F. Delouis, J. A. Delaire, D. Riehl, E. Anglaret, and F. Hache, "Picosecond and nanosecond polychromatic pump-probe studies of bubble growth in carbon nanotube suspensions," J. Opt. Soc. Am. B 19, 208-214 (2002).

14. R. C. Hollins, "Optical limiters: spatial, temporal and spectral effects," Nonlinear Opt. 21, 49-60 (1999).

15. P. T. Giovanneschi, P. A. Alloncle, D. Dufresne, Ph. Bournot, and M. Autric, "Study of the cavitation induced by a high power laser beam," in Seventh International Symposium on Gas Flow and Lasers, D. Schuoecker, ed., Proc. SPIE 1031, 545-550 (1989).

16. E. Goedert, R. Becker, A. Clements, and T. Whittaker III, "Time-resolved shadowgraphic imaging of the response of dilute suspensions to laser pulses," J. Opt. Soc. Am. B 15, 1442-1462 (1998).

17. C. Journet, W. K. Maser, P. Bernier, A. Loiseau, M. Lamy de la Chapelle, S. Lefrant, P. Deniard, R. Lee, and J. E. Fischer, "Large-scale production of single-walled carbon nanotubes by the electric-arc technique," Nature 388, 756-758 (1997).

18. L. Vaccarini, C. Goze, R. Aznar, V. Micholet, C. Journet, and P. Bernier, "Purification procedure of carbon nanotubes," Synth. Met. 103, 2492-2493 (1999).

19. S. Rols, R. Almairac, L. Henrard, E. Anglaret, and J. L. Sauvajol, "Diffraction by finite-size crystalline bundles of single wall nanotubes," Eur. Phys. J.B 10, 263-270 (1999).

20. L. Vivien, E. Anglaret, D. Riehl, F. Hache, F. Bacou, M. Andrieux, F. Lafonta, C. Journet, C. Goze, M. Brunet, and P. Bernier, "Optical limiting properties of singlewall carbon nanotubes," Opt. Commun. 174, 271-275 (2000). 